\title{
Quality of life in relation to tamoxifen or exemestane treatment in postmenopausal breast cancer patients: a Tamoxifen Exemestane Adjuvant Multinational (TEAM) Trial side study
}

\author{
J. G. H. van Nes - D. B. Y. Fontein - E. T. M. Hille $\cdot$ D. W. Voskuil $~$ \\ F. E. van Leeuwen $\cdot$ J. C. J. M. de Haes $\cdot$ H. Putter $\cdot$ C. Seynaeve $\cdot$ \\ J. W. R. Nortier $\cdot$ C. J. H. van de Velde
}

Received: 6 March 2012/ Accepted: 8 March 2012/Published online: 28 March 2012

(C) The Author(s) 2012. This article is published with open access at Springerlink.com

\begin{abstract}
Tamoxifen and aromatase inhibitors are associated with side effects which can significantly impact quality of life (QoL). We assessed QoL in the Tamoxifen Exemestane Adjuvant Multinational (TEAM) Trial and compared these data with reported adverse events in the main database. 2,754 Dutch postmenopausal early breast cancer patients were randomized between 5 years of exemestane, or tamoxifen (2.5-3 years) followed by exemestane (2.5-2 years). 742 patients were invited to participate in the QoL side study and complete questionnaires at 1 (T1) and 2 (T2) years after start of endocrine treatment. Questionnaires comprised the EORTC QLQ-C30 and BR23 questionnaires, supplemented with FACT-ES questions. 543 patients completed questionnaires at
\end{abstract}

This study is conducted on behalf of the Dutch TEAM Steering Committee

J. G. H. van Nes and D. B. Y. Fontein contributed equally to the study and the manuscript.

Partly presented as oral presentation at the 31rd San Antonio Breast Cancer Symposium, 2008, San Antonio, Texas.

J. G. H. van Nes - D. B. Y. Fontein - E. T. M. Hille ·

C. J. H. van de Velde $(\square)$

Department of Surgery, K6-R, Leiden University Medical

Centre, P.O. Box 9600, 2300, RC, Leiden, The Netherlands

e-mail: c.j.h.van_de_velde@lumc.nl

J. G. H. van Nes

Department of Radiotherapy, University Medical Center

Groningen, Groningen, The Netherlands

D. W. Voskuil · F. E. van Leeuwen

Department of Epidemiology, Netherlands Cancer Institute,

Amsterdam, The Netherlands

J. C. J. M. de Haes

Department of Psychology, Academic Medical Center,

University of Amsterdam, Amsterdam, The Netherlands
T1 and 454 patients $(84 \%)$ at T2. Overall QoL and most functioning scales improved over time. The only clinically relevant and statistically significant difference between treatment types concerned insomnia; exemestane-treated patients reported more insomnia than tamoxifen-treated patients. Discrepancy was observed between QoL issue scores reported by the patients and adverse events reported by physicians. Certain QoL issues are treatment- and/or time-specific and deserve attention by health care providers. There is a need for careful inquiry into QoL issues by those prescribing endocrine treatment to optimize QoL and treatment adherence.

Keywords Adverse effects - Breast cancer - Exemestane . Quality of life · Tamoxifen

\section{Background}

The majority of breast cancer patients are diagnosed at postmenopausal age and most have hormone receptor-

H. Putter

Department of Medical Statistics, Leiden University Medical Centre, Leiden, The Netherlands

C. Seynaeve

Department of Medical Oncology, Erasmus MC-Daniel den Hoed Cancer Center, Rotterdam, The Netherlands

J. W. R. Nortier

Department of Medical Oncology, Leiden University Medical

Centre, Leiden, The Netherlands 
positive tumors. Over time, adjuvant endocrine therapy has increasingly been used to reduce disease recurrence and improve survival [1]. Presently, optimal endocrine therapy consists of at least 5 years of treatment including an aromatase inhibitor (AI), either given upfront or as part of a sequential treatment regimen following tamoxifen [2]. Both regimens are appropriate treatment options for postmenopausal hormone receptor-positive breast cancer patients [2, 3]. However, many patients on endocrine therapy are confronted with adverse effects, which may negatively impact QoL, treatment compliance, and may then lead to a reduced survival $[4,5]$. The impact of longterm endocrine treatment on quality of life (QoL) in postmenopausal breast cancer patients may therefore be an important issue of deliberation regarding the choice for a specific adjuvant treatment strategy.

Both tamoxifen, a selective estrogen receptor modulator, and AIs, which potently inhibit the aromatase enzyme (involved in the conversion of androgens to estrogen), are associated with a variety of adverse effects. Tamoxifen is associated with thromboembolic complications and endometrial cancer while AIs show fewer life-threatening side effects but more readily give rise to sometimes invalidating symptoms such as hot flashes, arthralgias, vaginal dryness, and osteoporosis $[6,7]$. Variations in the types and severities of adverse effects associated with the use of either tamoxifen or an AI may result in differences in the domains of QoL affected in patients using either endocrine treatment.

So far, several trials have investigated QoL in patients using adjuvant endocrine therapy, but only four have compared QoL in patients treated with tamoxifen versus an AI [8-12]. It is difficult to compare these studies due to variations in trial design, starting time of the AI, and type of AI used. To the best of our knowledge, the ATAC QoL study is the only large trial that compared QoL from the start of endocrine therapy in patients treated with tamoxifen versus an AI upfront [9]. In the Tamoxifen Exemestane Adjuvant Multinational (TEAM) trial (Netherlands Trial Register NTR267), postmenopausal, hormone receptor-positive early breast cancer patients were randomized to either 5 years of exemestane upfront or 2.5-3 years tamoxifen followed by 2-2.5 years of exemestane [2]. There was a major participation in the TEAM study from the different hospitals throughout the Netherlands, therefore, this study provided a good opportunity for studying the effects of exemestane and tamoxifen on QoL in a homogeneous cohort of Dutch breast cancer patients. Moreover, we were able to relate relevant QoL issues reported by patients in this side study to the adverse events involved with these issues reported by the same patients in the main study using the registered adverse events.

\section{Patients and methods}

\section{Study design}

The study design and patient eligibility criteria for the TEAM trial have been described previously [2]. In the Netherlands, the study was initiated in 76 hospitals and details also have been described previously [13]. The TEAM QoL side study was an open multicenter study in which 45 Dutch TEAM centers participated. The side study protocol was approved separately by central and local ethics authorities before the enrollment of patients.

\section{Patients and data collection}

Patients who were randomized between January 2nd, 2003 and December 29th, 2004 and were event-free were invited to participate in the TEAM QoL side study. Patients received a letter together with the first QoL questionnaire at 1 year after treatment randomization (further referred to as time point 1; T1). Participating patients who returned the first questionnaire and were disease-free 2 years after randomization received the second questionnaire 1 year after T1 (further referred to as time point 2; T2). Patients included in the sequential arm received the second questionnaire before the switch from tamoxifen to exemestane. No questionnaire was sent at baseline (time of diagnosis and treatment) as the results regarding QoL may potentially be biased, due to the recent knowledge of breast cancer diagnosis and impending treatment, which is known to have a negative impact on QoL. Furthermore, treatment was allocated by randomization, hence there is essentially no indication for baseline imbalance in QoL data between both treatment arms [14]. Patient, tumor, treatment, and survival data were collected through the main TEAM Datacenter in Leiden, the Netherlands. In the main trial, patients were seen every 3 months in the first year, twice yearly in the second year and at least yearly thereafter. In the main trial, data on adverse events experienced by patients were recorded during follow-up visits by local investigators and centrally collected at the main datacenter. For the QoL participants, we selected adverse events reported within the first 2 years that were associated with the relevant QoL issues observed from the central database.

\section{Questionnaires}

Data on QoL were obtained using the European Organization for Research and Treatment of Cancer Quality of Life Questionnaire Version 3.0 (EORTC QLQ-C30) and the EORTC Breast Cancer Module questionnaire (QLQ-BR23), both translated into Dutch and previously validated [15, 16]. Both questionnaires were used after authorization by the EORTC 
Quality of Life Study Group. The EORTC QLQ-C30 is composed of five functioning scales (physical functioning, role functioning, cognitive functioning, emotional functioning, and social functioning), a global health status/QoL scale, three symptom scales (fatigue, pain, nausea/vomiting), and six single items (dyspnea, appetite loss, sleep disturbance, constipation, diarrhea, and financial impact). The EORTC QLQBR23 is a validated tool designed for breast cancer patients with varying disease stages and treatment modalities and consists of 23 items that assess disease symptoms, side effects, body image, sexual functioning, future perspectives, therapy side effects, breast and arm symptoms, and hair loss. Items that specifically assess side effects of chemotherapy were not applicable for the current study. In addition, the Functional Assessment of Cancer Therapy-Endocrine Subscale (FACTES) questionnaire was designed and validated to measure QoL in breast cancer patients treated with endocrine therapies [17]. Of the 18 items, 13 were included in our questionnaire (as other items were already included through the EORTC QLQ$\mathrm{C} 30$ or BR23 questionnaires), resulting in three endocrine symptom scales (menopausal complaints, weight complaints, and vaginal complaints).

Based on standard EORTC scoring procedures, all scales were linearly converted to a 0 -to-100 scale. Missing data were treated according to published recommendations [18]. For scales evaluating global health and functioning, higher scores represent higher levels of functioning and health status. For the evaluation of symptoms, higher scores correspond to more problems and higher levels of complaints.

\section{Relevant patient-reported outcomes}

Regarding QoL, the following items were investigated: (1) the difference between the QoL scores for patients using tamoxifen versus exemestane, (2) the difference between the two time points (T1 and T2), and (3) the interaction between treatment arm and time. A difference in score of at least eight points between groups was considered clinically relevant, and has been demonstrated to be a reasonable cutoff for clinical significance for a range of QoL endpoints [19]. Prior surgery was taken into account for analyses of body image, sexual functioning, and sexual enjoyment.

To study the association between the relevant QoL issues as reported by the patients and the related adverse events recorded for these patients by their treating physicians in the main database, patients whose questionnaire item scores were worse than the mean EORTC QLQ-C30 and BR23 reference scores were considered for comparison [20].

\section{Statistical analysis}

All data were analyzed using the statistical package SPSS for Windows 17.0 (SPSS Inc, Chicago, IL, USA). Descriptive data are given as mean (SD) or median (range). The $t$ test was used to compare frequencies between groups. Linear mixed models were used to assess changes over time for overall QoL and for separate components of QoL.

\section{Results}

Demographics

A total of 742 Dutch patients were invited to participate in the QoL side study (Fig. 1). Five-hundred-forty-three patients $(73 \%)$ completed the first questionnaire, of which $454(84 \%)$ also completed the second questionnaire.

Fig. 1 Patient selection

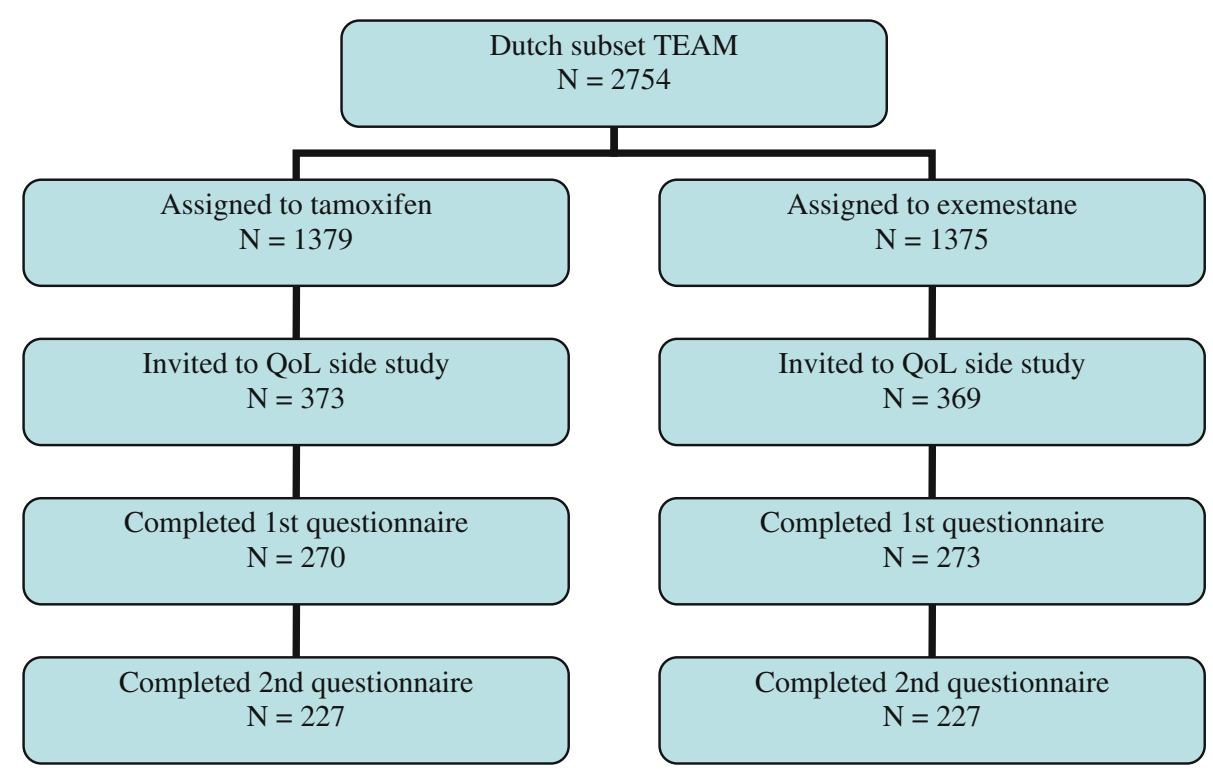


Baseline characteristics of the responding patients and the total group of Dutch TEAM patients are shown in Table 1. The distribution of clinicopathological and treatment characteristics of patients participating in the QoL side study was similar to that of the entire cohort of Dutch TEAM trial patients, except for the distribution of age, hormone receptor status, and prior chemotherapy (yes/no). Of the patients participating in the TEAM QoL side study, most were older than 60 years, had node-positive disease, and underwent a sentinel lymph node procedure followed by an axillary lymph node dissection. Almost $50 \%$ of the tumors were smaller than $20 \mathrm{~mm}$ and approximately half of the patients were treated by mastectomy.

\section{QoL: tamoxifen versus exemestane}

The results regarding QoL-items are shown in Table 2. In general, the scores for the various issues did not differ significantly between patients using tamoxifen versus exemestane. Patients allocated to tamoxifen showed superior scores for emotional functioning and sexual functioning ( $p=0.048$ and $p=0.024$ respectively) than exemestane users. Treatment with exemestane did not show superior results compared to tamoxifen for any of the functioning scales. Regarding individual symptoms, patients who received tamoxifen had fewer complaints of fatigue, dyspnea, insomnia, and arm symptoms than patients receiving exemestane. For "fatigue," the results were unrelated to the administration of chemotherapy (data not shown). Only for insomnia, the differences between the two treatment types were clinically significant (more than eight points difference between tamoxifen and exemestane), observed at both time points (Fig. 2). The endocrine symptom scales that were assessed using the FACT-ES included menopausal, weight, and vaginal complaints. These scores did not differ between treatment arms.

The global health status scale represents an overall summary measurement of QoL. With respect to either treatment group, there was no difference in global health status/overall QoL (Table 2). Interestingly, the reported overall QoL was higher than the reference value of the EORTC QLQ-C30 (>75 vs. 62 points).

\section{QoL: changes over time}

Changes in QoL items were assessed over the 1-year period between $\mathrm{T} 2$ and $\mathrm{T} 1$ for the total group of patients, as there were no relevant differences between the two treatment types. We found that over time, most functioning scales improved, except for physical functioning, sexual functioning, and sexual enjoyment $(p<0.01)$. Of note, fewer patients completed the questions concerning sexual functioning and enjoyment compared to the other items (data not shown). Over time, there was also no change in global health status; neither improvement nor deterioration. Concerning the individual symptom scales, a significant improvement was found for the following items: fatigue, nausea and vomiting, appetite, breast symptoms, and side effects of systemic therapy. Again, these results for fatigue were unrelated to the administration of chemotherapy (data not shown). A clinically significant difference over time was only established for breast symptoms.

QoL: interaction between treatment arm and time

Irrespective of treatment, most assessed items improved from $\mathrm{T} 1$ to $\mathrm{T} 2$. Only for the functioning scale "Future perspective" did an interaction exist between treatment and time: patients using exemestane improved more compared to patients using tamoxifen.

QoL compared to relevant adverse events issues reported in the TEAM trial

The QoL side study scores for sexual functioning and for sexual enjoyment were below the mean EORTC QLQ-C30 reference score for $58 \%$ of patients and $72 \%$ of patients, respectively, at T1; and values were similar at T2 [20]. In contrast, adverse events related to sexual functioning and/ or sexual enjoyment from the central database, including genital or vaginal discharge, decreased/loss of libido, vaginal dryness, and vulvovaginal disorders, were only documented for $3 \%$ of the QoL participants. Concerning insomnia, almost $60 \%$ of the QoL patients had a higher score compared to the mean reference score of the EORTC at $\mathrm{T} 1$ and $\mathrm{T} 2$ (indicating more sleeping problems), while in the central database, insomnia was recorded as adverse event by only $4 \%$ of the QoL study participants [20]. Lastly, fatigue was reported as adverse event by $12 \%$ of the QoL participants in the main TEAM database compared to $45 \%$ of QoL study patients having a higher score than the mean EORTC reference score for fatigue, indicating more complaints, observed at both T1 and T2 [20].

\section{Discussion}

The impact of adjuvant endocrine therapy on QoL is an ongoing discussion in the treatment of breast cancer patients prescribed long-term endocrine therapy. The current standard of practice advocating 5 or more years of endocrine treatment can therefore be considered cumbersome in those experiencing severe adverse effects. Both tamoxifen and AIs have been associated with the development of various menopausal symptoms like sleeping disorders and sexual problems related to the depletion of circulating estrogens, some of which being severe to the 
Table 1 Clinicopathological data of responders and all Dutch TEAM patients
$A L N D$ axillary lymph node dissection; $B C S$ breast conserving surgery; $E R$ estrogen receptor; $M S T$ mastectomy; $n p$ not performed;

$P g R$ progesterone receptor; $R T$ radiotherapy; $S L N P$ sentinel lymph node procedure;

TEAM NL all patients included in the Netherlands

\begin{tabular}{llllll}
\hline & \multicolumn{2}{l}{ Responders } & & TEAM NL & $p$-value \\
\cline { 2 - 3 } & $N$ & & N
\end{tabular}

\begin{tabular}{lllll}
\hline Total & 543 & 100 & 2,753 & 100
\end{tabular}

Age

$<50-59$

$60-69$

200

37

914

33

965

0.039

$\geq 70$

143

37

26

874

35

Body mass index

$\leq 25$

190

39

919

931

601

0.589

$\geq 30$

109

39

22

Pathological tumor stage

T1

267

$\mathrm{T} 2$

241

$\mathrm{T} 3$ and $\mathrm{T} 4$

32

Pathological nodal stage

$\begin{array}{lr}\mathrm{pN} 0 & 150 \\ \mathrm{pN} 1-3 & 275 \\ \mathrm{pN} 4-9 & 77 \\ \mathrm{pN} \geq 10 & 18\end{array}$

Histological grade

Grade I

Grade II

Grade III

179

Type of tumor

Ductal

404

Lobular

Ductal lobular

Other

49

32

45

6

38

38

25

77

18

15

3

17

35

Hormone receptor

$\mathrm{ER}+, \mathrm{PgR}+$

ER+, PgRnp

ER-, PgR+

ER-, PgR-

Local therapy

MST, RT-

MST, RT+

BCS, RT-

BCS, RT+

Treatment axilla

SLNP-, ALND- 0

SLNP-, ALND+ 172

SLNP+, ALND- $\quad 127$

SLNP+, ALND+

244

Chemotherapy

$\begin{array}{ll}\text { No } & 348 \\ \text { Yes } & 195\end{array}$

1,235

1,329

183

834

1,387

327

131

420

1,218

934

45
48
7

31

52

12

12

5

16

47

36

2,047

75

0.891

442

129

109

16

5

4

0.158

0.166

0.896

$1,233 \quad 45$
30
1,950

71

595

153

47

64
36

1,94

71

30

$1,127 \quad 41$

0.051

$401 \quad 15$

$36 \quad 1$

$1,188 \quad 43$

0.882

$632 \quad 23$

812

0.001

$$
6
$$$$
2
$$$$
0
$$

(1)

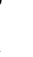

(1)

0

32

45

195

$\begin{array}{ll}1,941 & 71\end{array}$

0.002 
Table 2 Overview of the different functioning and symptom scales by time and treatment arm

\begin{tabular}{|c|c|c|c|c|c|c|c|}
\hline & \multicolumn{2}{|l|}{$\mathrm{T} 1$} & \multicolumn{2}{|l|}{$\mathrm{T} 2$} & \multicolumn{3}{|l|}{$p$-value } \\
\hline & $\begin{array}{l}\text { Tamoxifen } \\
\text { Mean (SD) }\end{array}$ & $\begin{array}{l}\text { Exemestane } \\
\text { Mean (SD) }\end{array}$ & $\begin{array}{l}\text { Tamoxifen } \\
\text { Mean (SD) }\end{array}$ & $\begin{array}{l}\text { Exemestane } \\
\text { Mean (SD) }\end{array}$ & Treatment & Time & Time by treatment \\
\hline \multicolumn{8}{|l|}{ EORTC QLQ-C30 } \\
\hline \multicolumn{8}{|l|}{ Functioning scales } \\
\hline Physical functioning & $80(18)$ & $78(18)$ & 79 (18) & 79 (17) & 0.732 & 0.508 & 0.132 \\
\hline Role functioning & $80(25)$ & $79(28)$ & $82(26)$ & $82(25)$ & 0.741 & 0.028 & 0.826 \\
\hline Cognitive functioning & $83(21)$ & $79(25)$ & 85 (19) & $83(22)$ & 0.082 & 0.002 & 0.179 \\
\hline Emotional functioning & $80(21)$ & $75(21)$ & $83(20)$ & $81(21)$ & 0.048 & $<0.001$ & 0.273 \\
\hline Social functioning & $87(20)$ & $86(19)$ & $90(19)$ & $90(19)$ & 0.861 & 0.001 & 0.397 \\
\hline \multicolumn{8}{|l|}{ Global health scale } \\
\hline Global health status & $78(18)$ & 75 (19) & $78(17)$ & $76(17)$ & 0.074 & 0.458 & 0.281 \\
\hline \multicolumn{8}{|l|}{ Symptom scales } \\
\hline Fatigue & $30(25)$ & $34(26)$ & $24(23)$ & $29(23)$ & 0.026 & $<0.001$ & 0.661 \\
\hline Pain & $20(24)$ & $21(25)$ & $18(24)$ & $20(25)$ & 0.216 & 0.234 & 0.643 \\
\hline Nausea and vomiting & $6(15)$ & $6(17)$ & $4(14)$ & $3(14)$ & 0.917 & 0.004 & 0.395 \\
\hline \multicolumn{8}{|l|}{ Symptom single items } \\
\hline Dyspnea & $15(24)$ & $20(25)$ & $14(23)$ & $18(24)$ & 0.032 & 0.234 & 0.490 \\
\hline Appetite loss & $9(22)$ & $9(19)$ & $5(16)$ & $6(15)$ & 0.697 & 0.001 & 0.511 \\
\hline Insomnia & $28(32)$ & $37(31)$ & $27(30)$ & $35(31)$ & 0.001 & 0.188 & 0.869 \\
\hline Constipation & $12(24)$ & $12(23)$ & $13(24)$ & $10(55)$ & 0.319 & 0.507 & 0.337 \\
\hline Diarrhea & $4(15)$ & $6(17)$ & $4(15)$ & $5(15)$ & 0.236 & 0.458 & 0.823 \\
\hline Financial difficulties & $8(18)$ & $5(15)$ & $9(22)$ & $6(16)$ & 0.076 & 0.431 & 0.791 \\
\hline \multicolumn{8}{|l|}{ EORTC QLQ-B23 } \\
\hline \multicolumn{8}{|l|}{ Functioning scales } \\
\hline Body image $^{\mathrm{a}}$ & $84(25)$ & $83(22)$ & $87(20)$ & $84(26)$ & 0.327 & 0.004 & 0.294 \\
\hline Sexual functioning ${ }^{\mathrm{a}}$ & $20(19)$ & $17(19)$ & $21(19)$ & $16(19)$ & 0.024 & 0.755 & 0.208 \\
\hline Sexual enjoyment ${ }^{\mathrm{a}}$ & $50(30)$ & $46(23)$ & $48(22)$ & $44(22)$ & 0.172 & 0.162 & 0.829 \\
\hline Future perspective & $67(27)$ & $64(26)$ & $70(25)$ & $72(25)$ & 0.710 & $<0.001$ & 0.028 \\
\hline \multicolumn{8}{|l|}{ Symptom scales } \\
\hline Syst therapy side effect & $19(17)$ & $19(18)$ & $17(17)$ & $18(17)$ & 0.964 & 0.040 & 0.426 \\
\hline Breast symptoms & $22(19)$ & $19(19)$ & $16(19)$ & $14(19)$ & 0.152 & $<0.001$ & 0.523 \\
\hline Arm symptoms ${ }^{\mathrm{b}}$ & $19(21)$ & $17(21)$ & $20(20)$ & $16(20)$ & 0.027 & 0.728 & 0.213 \\
\hline \multicolumn{8}{|l|}{ EORTC FACT-ES } \\
\hline Menopausal complaints & $26(21)$ & $26(21)$ & $24(20)$ & $26(20)$ & 0.572 & 0.093 & 0.196 \\
\hline Weight complaints & $14(20)$ & $16(20)$ & 15 (19) & $16(19)$ & 0.186 & 0.207 & 0.972 \\
\hline Vaginal complaints & $18(27)$ & $20(27)$ & $20(26)$ & $23(26)$ & 0.337 & 0.412 & 0.793 \\
\hline
\end{tabular}

${ }^{a}$ For this analysis, surgery was a stratification factor: mastectomy versus breast conserving surgery

${ }^{\mathrm{b}}$ For this analysis, surgery was a stratification factor: axillary lymph node dissection (no/yes)

point of significantly diminishing QoL. The present investigation of QoL in patients in the TEAM trial offers further insight into the impact of either tamoxifen or exemestane on a woman's QoL during endocrine therapy for breast cancer.

In the present investigation, a clinically significant difference was found between the two treatment arms for insomnia, observed at both time points, indicating more problems for exemestane users versus those taking tamoxifen. In general, insomnia is underreported and frequently overlooked in the context of breast cancer treatment. Approximately, half of all breast cancer patients experience sleeping disorders up to several years postdiagnosis [21]. The pathophysiological mechanism behind insomnia in breast cancer patients suggests a relation with nocturnal hot flashes [22]. Both hot flashes and musculoskeletal symptoms have also been associated with the depletion of circulating estrogens [23]. As exemplified by 


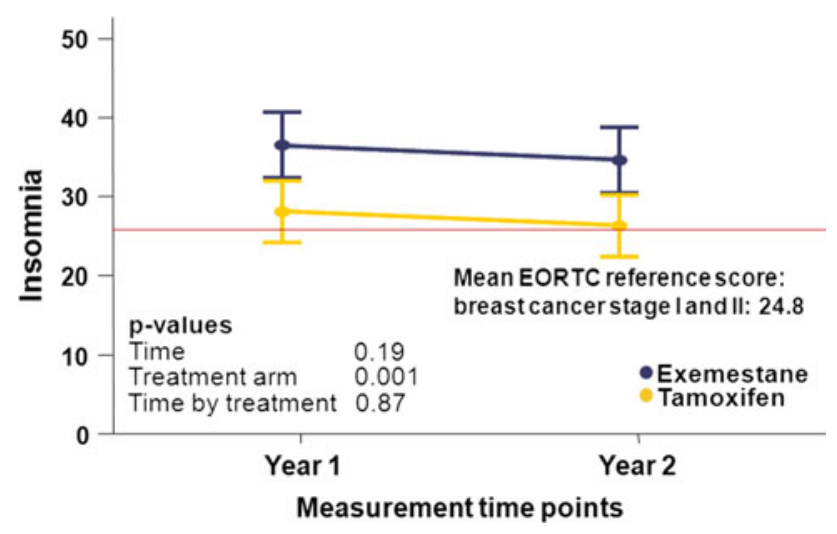

Fig. 2 Insomnia in relation to treatment and time in the TEAM QoL side study

the MA.17 trial, a significant increase in the incidence of hot flashes and musculoskeletal symptoms was found in patients treated with letrozole compared to placebo [11]. Our data regarding more sleeping disorders in exemestane users suggests that further lowering of postmenopausal estrogen levels with exemestane may lead to more sleeping disorders. Unfortunately, this cannot be verified with blood samples, as these were not collected for our cohort of TEAM patients.

Patients using exemestane reported less sexual enjoyment and more sexual functioning problems than patients using tamoxifen. This is similar to the results as found after 1 year of therapy in the US Oncology side study of the TEAM trial concerning menopausal symptoms [10]. Our data do show that also after 2-2.5 years of therapy, menopausal symptoms persisted over time. In physiological menopause, the lack of circulating estrogens reduces vaginal lubrication, resulting in vaginal dryness and, consequently, dyspareunia [24]. Tamoxifen affects sexual functioning in terms of decreased libido and the ability to become aroused and experience orgasm, while AIs cause vaginal dryness and dyspareunia. Although tamoxifen is known to have anti-estrogenic properties on breast tissue, it exerts an estrogen agonist effect on the female genital tract in postmenopausal women and increases the risk of endometrial cancer [25]. Furthermore, under tamoxifen treatment, the vaginal squamous epithelium is weakly stimulated and undergoes proliferation and maturation [26]. It is possible that the abovementioned reasons explain why sexual functioning may be less affected in tamoxifentreated patients than in those treated with exemestane. Another contributing factor may be that as already said, exemestane induces further lowering of postmenopausal estrogens in breast cancer patients. Fewer reports investigated vaginal dryness and dyspareunia in studies with AIs, but Morales suggest that AIs induce more symptoms of vaginal atrophy (vaginal dryness and dyspareunia) than tamoxifen, which parallels our findings that exemestanetreated patients reported more sexual functioning problems than tamoxifen-treated patients [27].

Adverse events and reported QoL

Although it is difficult to relate QoL issues as measured with questionnaires with adverse events as documented by the physician, we observed striking differences between these two methods. With respect to specific aspects of QoL such as sexual functioning, fatigue, and insomnia, significantly more patients reported complaints of these items in the QoL side study than that adverse events related to these specific complaints were documented in the main TEAM trial database. This finding reiterates the importance of thorough investigations on QoL issues and questions the reliability of the reported adverse events in large multinational phase III trials. Ideally, every large clinical trial assessing efficacy and safety of new oncological treatments should include a questionnaire-based QoL assessment, enabling more precise estimation of the associated adverse events.

\section{Other QoL studies}

To date, only a few large randomized trials comparing adjuvant tamoxifen with an AI have reported on QoL data (Table 3) [8-12]. Also, it is difficult to compare the different randomized trials with each other and with our QoL side study, due to differences in patient populations, countries of residence, AIs used, timing and of start of treatment, and the instruments used to assess QoL. However, regardless of these variations, no large differences in QoL were seen between tamoxifen and AIs.

The planned nature of the QoL side study using validated questionnaires as well as the high response rate for both T1 and T2 questionnaires lends confidence to our findings. The absence of a baseline measurement may be considered a shortcoming when assessing changes in QoL over time; notably, however, baseline measurements of QoL are likely biased due to recent knowledge of breast cancer diagnosis in our patient population at the start of treatment. Due to the randomized nature of this trial, differences in baseline QoL with respect to treatment arms are unlikely [14]. This study is limited by the lack of reporting consistency of adverse events in the main TEAM trial in relation to the observed QoL domains affected in patients in the side study. Concurrently, this may still adequately reflect variations in reporting by both investigators and patients alike during clinical visits. 


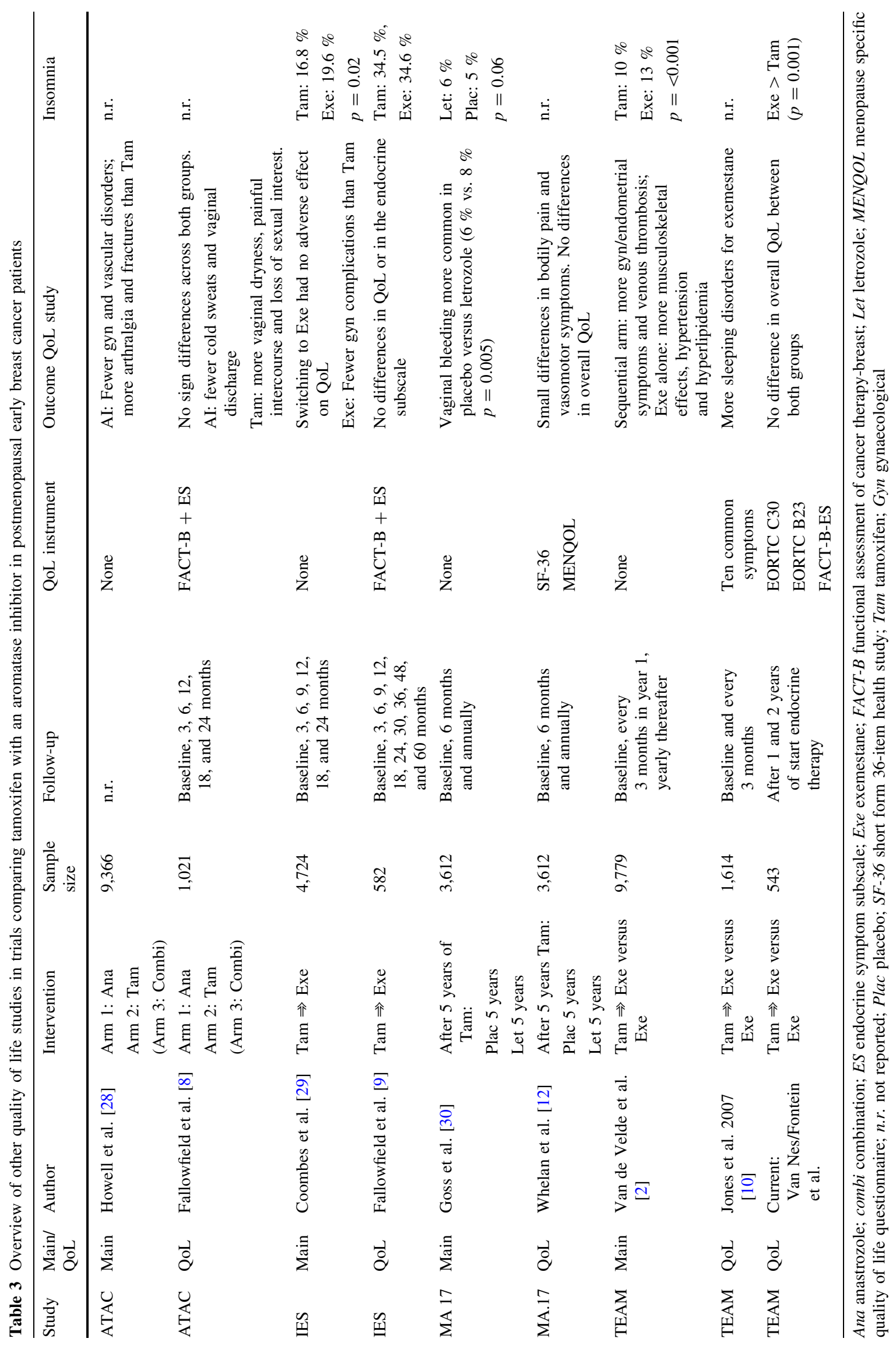




\section{Conclusion}

Our findings indicate that overall QoL and most functioning scales improve with longer therapy duration, both for patients treated with tamoxifen and exemestane. Nevertheless, certain QoL issues are treatment-specific and deserve attention by oncology health care providers. Also, the large number of patients who reported complaints of sexual functioning, fatigue, and insomnia in the QoL study was not mirrored by the reported adverse events related to these complaints in the main TEAM trial database. Although strictly observational, this large discrepancy between various QoL issues in the side study and the related adverse events recorded in the main trial stresses the need for careful inquiry by those seeing patients throughout the duration of endocrine treatment to optimize QoL and ensure adherence to treatment. Further investigation into an optimal reporting approach is warranted.

Acknowledgments We are indebted to the women who participated in the Life style study of the TEAM trial. We also thank the Independent Monitoring Committee, the trial Steering Committee and the doctors, monitors, nurses, data managers, and other support staff for their participation in this trial. We thank Janneke Willems for study and administrative support and Wieke Heideman for data entry. We thank Jan Junggeburt, Elma Meershoek-Klein Kranenbarg en Marjolijn Duijm-de Carpentier of the Central Datacenter. We thank the EORTC Quality of Life Group for using their questionnaires. The TEAM Life style study is supported by a grant of the Dutch Pink Ribbon Foundation and by an unrestricted research grant from Pfizer.

Conflict of interest The authors have declared no conflicts of interest.

Open Access This article is distributed under the terms of the Creative Commons Attribution License which permits any use, distribution, and reproduction in any medium, provided the original author(s) and the source are credited.

\section{References}

1. Davies C, Godwin J, Gray R et al (2011) Relevance of breast cancer hormone receptors and other factors to the efficacy of adjuvant tamoxifen: patient-level meta-analysis of randomised trials. Lancet 378:771-784

2. van de Velde CJ, Rea D, Seynaeve C et al (2011) Adjuvant tamoxifen and exemestane in early breast cancer (TEAM): a randomised phase 3 trial. Lancet 377:321-331

3. Regan MM, Neven P, Giobbie-Hurder A et al (2011) Assessment of letrozole and tamoxifen alone and in sequence for postmenopausal women with steroid hormone receptor-positive breast cancer: the BIG 1-98 randomised clinical trial at 8.1 years median follow-up. Lancet Oncol 12:1101-1108

4. Mortimer JE, Flatt SW, Parker BA et al (2008) Tamoxifen, hot flashes and recurrence in breast cancer. Breast Cancer Res Treat 108:421-426

5. Patterson RE, Saquib N, Natarajan L et al (2011) Improvement in self-reported physical health predicts longer survival among women with a history of breast cancer. Breast Cancer Res Treat 127:541-547
6. Burstein HJ (2007) Aromatase inhibitor-associated arthralgia syndrome. Breast 16:223-234

7. Fisher B, Costantino JP, Wickerham DL et al (2005) Tamoxifen for the prevention of breast cancer: current status of the National Surgical Adjuvant Breast and Bowel Project P-1 study. J Natl Cancer Inst 97:1652-1662

8. Fallowfield L, Cella D, Cuzick J et al (2004) Quality of life of postmenopausal women in the Arimidex, Tamoxifen, Alone or in Combination (ATAC) Adjuvant Breast Cancer Trial. J Clin Oncol 22:4261-4271

9. Fallowfield LJ, Bliss JM, Porter LS et al (2006) Quality of life in the intergroup exemestane study: a randomized trial of exemestane versus continued tamoxifen after 2 to 3 years of tamoxifen in postmenopausal women with primary breast cancer. J Clin Oncol 24:910-917

10. Jones SE, Cantrell J, Vukelja S et al (2007) Comparison of menopausal symptoms during the first year of adjuvant therapy with either exemestane or tamoxifen in early breast cancer: report of a Tamoxifen Exemestane Adjuvant Multicenter trial substudy. J Clin Oncol 25:4765-4771

11. Muss HB, Tu D, Ingle JN et al (2008) Efficacy, toxicity, and quality of life in older women with early-stage breast cancer treated with letrozole or placebo after 5 years of tamoxifen: NCIC CTG intergroup trial MA.17. J Clin Oncol 26:1956-1964

12. Whelan TJ, Goss PE, Ingle JN et al (2005) Assessment of quality of life in MA.17: a randomized, placebo-controlled trial of letrozole after 5 years of tamoxifen in postmenopausal women. J Clin Oncol 23:6931-6940

13. van Nes JGH, Seynaeve C, Maartense E et al (2010) Patterns of care in Dutch postmenopausal patients with hormone-sensitive early breast cancer participating in the Tamoxifen Exemestane Adjuvant Multinational (TEAM) trial. Ann Oncol 21:974-982

14. Fayers P, King M (2008) The baseline characteristics did not differ significantly. Qual Life Res 17:1047-1048

15. Aaronson NK, Ahmedzai S, Bergman B et al (1993) The European Organization for Research and Treatment of Cancer QLQC30: a quality-of-life instrument for use in international clinical trials in oncology. J Natl Cancer Inst 85:365-376

16. Sprangers MA, Groenvold M, Arraras JI et al (1996) The European Organization for Research and Treatment of Cancer breast cancer-specific quality-of-life questionnaire module: first results from a three-country field study. J Clin Oncol 14: 2756-2768

17. Fallowfield LJ, Leaity SK, Howell A et al (1999) Assessment of quality of life in women undergoing hormonal therapy for breast cancer: validation of an endocrine symptom subscale for the FACT-B. Breast Cancer Res Treat 55:189-199

18. Fayers PM (2001) Interpreting quality of life data: populationbased reference data for the EORTC QLQ-C30. Eur J Cancer 37:1331-1334

19. Norman GR, Sloan JA, Wyrwich KW (2003) Interpretation of changes in health-related quality of life: the remarkable universality of half a standard deviation. Med Care 41:582-592

20. Scott NW, Fayers PM, Aaronson NK et al (2011) EORTC QLQC30 reference values. EORTC, Brussels

21. Savard J, Simard S, Blanchet J et al (2001) Prevalence, clinical characteristics, and risk factors for insomnia in the context of breast cancer. Sleep 24:583-590

22. Ohayon MM (2006) Severe hot flashes are associated with chronic insomnia. Arch Intern Med 166:1262-1268

23. Stearns V, Ullmer L, Lopez JF et al (2002) Hot flushes. Lancet 360:1851-1861

24. Semmens JP, Wagner G (1982) Estrogen deprivation and vaginal function in postmenopausal women. JAMA 248:445-448

25. Nasu K, Takai N, Nishida M et al (2008) Tumorigenic effects of tamoxifen on the female genital tract. Clin Med Pathol 1:17-34 
26. Varras M, Polyzos D, Akrivis C (2003) Effects of tamoxifen on the human female genital tract: review of the literature. Eur $\mathbf{J}$ Gynaecol Oncol 24:258-268

27. Morales L, Neven P, Timmerman D et al (2004) Acute effects of tamoxifen and third-generation aromatase inhibitors on menopausal symptoms of breast cancer patients. Anticancer Drugs 15:753-760

28. Howell A, Cuzick J, Baum M et al (2005) Results of the ATAC (Arimidex, Tamoxifen, Alone or in Combination) trial after completion of 5 years' adjuvant treatment for breast cancer. Lancet 365:60-62
29. Coombes RC, Kilburn LS, Snowdon CF et al (2007) Survival and safety of exemestane versus tamoxifen after 2-3 years' tamoxifen treatment (Intergroup Exemestane Study): a randomised controlled trial. Lancet 369:559-570

30. Goss PE, Ingle JN, Martino S et al (2005) Randomized trial of letrozole following tamoxifen as extended adjuvant therapy in receptor-positive breast cancer: updated findings from NCIC CTG MA.17. J Natl Cancer Inst 97:1262-1271 